\title{
Predictive Factors of Brain Death in Acute Neurocritical Patients Identified as Potential Organ Donors
}

\author{
Guixing $X u^{1,3 \#}$, Ping $\mathrm{Xu}^{2 \#}$, Jing Zhao ${ }^{2 \#}$, Zhiyong Guo ${ }^{1 *}$ and Xiaoshun $\mathrm{He}^{1 *}$ \\ ${ }^{1}$ Organ Transplant Center, the First Affiliated Hospital, Sun Yat-sen University, China \\ ${ }^{2}$ Department of radiology, the First Affiliated Hospital, Sun Yat-sen University, China \\ ${ }^{3}$ Department of neurosurgery, the First Affiliated Hospital, Sun Yat-sen University, China \\ "These authors contributed equally to this work and should be considered as co-first authors
}

${ }^{*}$ Corresponding authors: Xiaoshun He and Zhiyong Guo, Organ Transplant Center, the First Affiliated Hospital, Sun Yat-sen University, NO.58 Zhongshan Er Road, Guangzhou, China, Tel: 86-20-82393181, Fax: 86-20-87306082, E-mail:xguix1981@163.com and gdtrc@126.com

\begin{abstract}
Objective: Reliable prediction of brain death after no-therapy decision in acute neurocritical patients is of specific interest for organ donation after brain death (DBD). We aimed to establish a neurological scoring system (the BD-N score) to predict the probability of progression to brain death after spontaneous respiratory arrest.

Methods: In this prospective observational study, we collected clinical data of acute neurocritical patients with a potential progression to BD, who was admitted in the First Affiliated Hospital of Sun Yet-sen University from May 2015 to April 2016. Final endpoint was adjudicated brain death (BD). The clinical data were compared between patients who identified as BD within 7 days and those who identified beyond 7 days, the time of spontaneous respiratory arrest as start point. Neurological examination, laboratory and radiographic data before the $\mathrm{BD}$, were recorded. We used univariate and multivariable logistic regression analyses to assess associations between brain death within 7 days after spontaneous respiratory arrest and these variables. Points attributed to each variable were summed to create a predictive score for BD in neurocritical patients (the BD-N score). We assessed performance of the score using receiver operating curve analysis.
\end{abstract}

Results: A total of 104 patients satisfied our inclusion criteria. $29(27.9 \%)$ patients identified as BD within 3-5 days, $50(48.1 \%)$ patients identified as BD within 5-7 days, 25 (24\%) patients identified as BD after 7 days. Multivariable logistic regression analysis showed that absent pupil reflex $(\mathrm{OR}=7.43,95 \% \mathrm{Cl} 1.62$ $33.99, \mathrm{P}=0.010)$, anisocoria or bilaterally dilated pupil $(\mathrm{OR}=$ $6.25,95 \% \mathrm{Cl} 1.21-32.22, \mathrm{P}=0.028$ ), absent motor response to pain $(\mathrm{OR}=15.89,95 \% \mathrm{Cl} 3.04-82.93, \mathrm{P}=0.001)$, absent cisterna ambiens $(\mathrm{OR}=11.96,95 \% \mathrm{Cl} 1.59-89.78, \mathrm{P}=0.016)$ and present of brain herniation $(\mathrm{OR}=16.28,95 \% \mathrm{Cl} 2.25-117.73, \mathrm{P}=0.006)$ on brain CT imagine were associated with brain death within 7 days. AUC for the score was $83.9 \%$ for prediction of brain death within 7 days. A score of $3-5$ was translated into an $81.9 \%$ chance (positive predictive value) of brain death within 7 days and a score of 1-2 translated into a $80 \%$ chance (negative predictive value) of brain death beyond 9 days.
Conclusion: The BD-N score can be used to predict time of brain death after spontaneous respiratory arrest in acute neurocritical patients. However, further prospective validation is needed.

\section{Keywords}

Neurocritical patients, Brain death, Prediction, Donor

\section{Abbreviations}

DBD: donation after brain death; CT: computed tomography; BD: brain death; TBI: traumatic brain injury

\section{Introduction}

Donation after brain death (DBD) protocols allows families of patients who are brain dead to donate organs. Such protocols have been implemented in many countries and might reduce the shortage of available organs for transplantation. Although such donation contributes an increasing proportion of viable organs for transplantation, identification of appropriate candidates is a restricting factor $[1,2]$.

Patients with catastrophic, irreversible brain injury named neurocritical patients, mainly included traumatic brain injury (TBI) and intracranial haemorrhage [1]. Neurocritical patients are most likely to progress to brain death (BD), so they were identified as potential organ donor, currently [2]. This type of potential organ donors requires intensive care unit (ICU) management to preserve organs until BD was confirmed. Nowadays, brain injury severity remains a subjective notion and is based on the clinicians' experience taking into account all the clinical and radiological signs, which could predict a life-threatening condition for the patient. Objective tools to better define severity are required, all the more so in the case of a potential progression to $\mathrm{BD}$.

Previous studies have identified some predictive factors for BD in comatose patients but only following intracranial haemorrhage or traumatic brain injuries which are not representative of neurocritical patients identified as potential organ donors [3-5]. However, 
identifying neurocritical patients who could progress to BD after notherapy decision is particularly difficult at the acute phase because of a lack of clearly established predictive factors. In clinical practice, the "no-therapy decision" is hard to define since the contents of no-therapy decision in each medical center were different. So we designed a study to identify predictive factors to establish the BD-N score (a neurological scoring system) to predict the probability of $\mathrm{BD}$ in acute neurocritical patients, after spontaneous respiratory arrest.

\section{Methods}

\section{Study design and participants}

In this observational study, we prospectively obtained data from consecutive adult, neurocritical patients after spontaneous respiratory arrest and a likely prognosis of progression to $\mathrm{BD}$ in the First Affiliated Hospital, Sun Yat-sen University, between 1 May 2015 and 30 April 2016.
The inclusion criteria were an apneic patient with deep coma and the presence of a recent spontaneous intracranial haemorrhage or traumatic brain injury on brain CT scan. We excluded patients without tracheal intubation or who suffered reversible coma. We selected variables for data collection on the basis of findings from existing study and our previous comprehensive analysis [4-6].

The baseline characteristics for this study included age, sex, personal cardiovascular risk factors (high blood pressure, diabetes, dyslipidemia and atrial fibrillation), diagnoses, and Glasgow Coma Scale (GCS) score. In order to exclude the bias of confounding medications, or prior use of illegal drugs or alcohol; a reasonable guideline is to calculate 5 to 7 times the drug's elimination half-life in hours and allow that time to pass before clinical examination is performed. Neurological examination after spontaneous respiratory arrest mainly focused on brain stem reflexes, such as pupil size, corneal reflex, cough reflex, motor response to pain (facial pain stimulation) and so on. The last brain CT scan imaging after spontaneous

Table 1: Clinical and demographic characteristics of patients.

\begin{tabular}{|c|c|c|c|c|c|}
\hline \multirow[b]{2}{*}{ Factors } & \multicolumn{4}{|c|}{ Groups (Brain death time) } & \multirow[b]{2}{*}{$P$} \\
\hline & $\begin{array}{l}\text { 3-5d group } \\
(n=29)\end{array}$ & $\begin{array}{l}\text { 5-7d group } \\
(n=50)\end{array}$ & $\begin{array}{l}>7 d \text { group } \\
(n=25)\end{array}$ & $\begin{array}{l}\text { All patients } \\
(n=104)\end{array}$ & \\
\hline \multicolumn{6}{|c|}{ Demographic characteristics } \\
\hline Age(years) & $40.10 \pm 14.08$ & $38.60 \pm 11.33$ & $45.96 \pm 9.28$ & $40.79 \pm 12.00$ & 0.039 \\
\hline days & $9.14 \pm 5.00$ & $5.52 \pm 3.48$ & $6.96 \pm 5.71$ & $6.88 \pm 4.74$ & 0.004 \\
\hline \multicolumn{6}{|l|}{ Gender } \\
\hline Male & $23(79.30)$ & $41(82.00)$ & $21(84.00)$ & $85(81.70)$ & 0.904 \\
\hline Female & $6(20.70)$ & $9(18.00)$ & $4(16.00)$ & $19(18.30)$ & \\
\hline \multicolumn{6}{|c|}{ Clinical characteristics } \\
\hline \multicolumn{6}{|l|}{ Diagnosis } \\
\hline hemorrhagic & $14(48.30)$ & $22(44.00)$ & $15(60.00)$ & $51(49.00)$ & 0.424 \\
\hline traumatic & $15(51.70)$ & $28(56.00)$ & $10(40.00)$ & $53(51.00)$ & \\
\hline \multicolumn{6}{|l|}{ Pupil shape } \\
\hline normal & $0(0.00)$ & $11(22.00)$ & $11(44.00)$ & $22(21.20)$ & $<0.001$ \\
\hline anisocoria & $3(10.30)$ & $11(22.00)$ & $8(32.00)$ & $22(21.200$ & \\
\hline mydriasis & $26(89.70)$ & $28(56.00)$ & $6(24.00)$ & $60(57.70)$ & \\
\hline \multicolumn{6}{|c|}{ Pupillary light reflex } \\
\hline Sensitive & $1(3.40)$ & $8(16.00)$ & $8(32.00)$ & $17(16.30)$ & $<0.001$ \\
\hline Slow & $2(6.90)$ & $17(34.00)$ & $11(44.00)$ & $30(28.80)$ & \\
\hline disappear & $26(89.70)$ & $25(50.00)$ & $6(24.00)$ & $57(54.80)$ & \\
\hline \multicolumn{6}{|l|}{ Corneal reflex } \\
\hline Sensitive & $0(0.00)$ & $5(10.00)$ & $9(36.00)$ & $14(13.50)$ & $<0.001$ \\
\hline disappear & $29(100.00)$ & $45(90.00)$ & $16(64.00)$ & $90(86.50)$ & \\
\hline \multicolumn{6}{|l|}{ Cough reflex } \\
\hline Yes & $1(3.40)$ & $6(12.00)$ & $13(52.00)$ & $20(19.20)$ & $<0.001$ \\
\hline no & $28(96.60)$ & $44(88.00)$ & $12(48.00)$ & $84(80.80)$ & \\
\hline \multicolumn{6}{|l|}{ Pain reaction } \\
\hline Sensitive & $0(0.00)$ & $2(4.00)$ & $5(20.00)$ & $7(6.70)$ & $<0.001$ \\
\hline Slow & $4(13.80)$ & $24(48.00)$ & $16(64.00)$ & $44(42.30)$ & \\
\hline disappear & $25(86.20)$ & $24(48.00)$ & $4(16.00)$ & $53(51.00)$ & \\
\hline \multicolumn{6}{|c|}{ Cisterna ambiens } \\
\hline Normal & $0(0.00)$ & $1(2.00)$ & $5(20.00)$ & $6(5.80)$ & $<0.001$ \\
\hline Narrow & $3(10.30)$ & $19(38.00)$ & $13(52.00)$ & $35(33.70)$ & \\
\hline disappear & $26(89.70)$ & $30(60.00)$ & $7(28.00)$ & $63(60.60)$ & \\
\hline \multicolumn{6}{|l|}{ Whirlpool sign } \\
\hline No & $11(37.90)$ & $21(42.00)$ & $15(60.00)$ & $47(45.20)$ & 0.219 \\
\hline Yes & $18(62.10)$ & $29(58.00)$ & $10(40.00)$ & $57(54.80)$ & \\
\hline \multicolumn{6}{|c|}{ Cerebral hernia } \\
\hline No & $3(10.30)$ & $19(38.00)$ & $15(60.00)$ & $37(35.60)$ & 0.001 \\
\hline Yes & $26(89.70)$ & $31(62.00)$ & $10(40.00)$ & $67(64.40)$ & \\
\hline \multicolumn{6}{|c|}{ Intraventricular hemorrhage } \\
\hline No & $5(17.20)$ & $22(44.00)$ & $17(68.00)$ & $44(42.30)$ & 0.001 \\
\hline Yes & $24(82.80)$ & $28(56.00)$ & $8(32.00)$ & $60(57.70)$ & \\
\hline \multicolumn{6}{|l|}{ Hydrocephalus } \\
\hline No & $25(86.20)$ & $42(84.00)$ & $21(84.00)$ & $88(84.60)$ & 0.962 \\
\hline Yes & $4(13.80)$ & $8(16.00)$ & $4(16.00)$ & $16(15.40)$ & \\
\hline \multicolumn{6}{|c|}{ Subarachnoid hemorrhage } \\
\hline No & $13(44.80)$ & $17(34.00)$ & $13(52.00)$ & $43(41.30)$ & 0.297 \\
\hline Yes & $16(55.20)$ & $33(66.00)$ & $12(48.00)$ & $61(58.70)$ & \\
\hline
\end{tabular}


respiratory arrest were similarly collected and transferred to the Neurosurgery Department of the First Affiliated Hospital, Sun Yatsen University. Type of CT scan imaging included parenchymal haematoma, subarachnoid haemorrhage, haematoma volume, cisterna ambiens, evidence of herniation (the shift of midline was the typical manifestation), presence of intraventricular haemorrhage and the presence of hydrocephalus. The images were systematically inspected by radiologists and neurosurgeons who participated in the study. Hydrocephalus was defined as increased radius or decreased ventricular angle in frontal horns, rounding and enlargement of atrium with sulcal effacement, increased width of third ventricle, or ballooning of fourth ventricle [5,6]. Clinicians analysing the CT data were kept unaware of the patient's status (BD or not).

After inclusion in the study, patients were followed up until $\mathrm{BD}$ was confirmed. Each case of BD was confirmed by two medical clinical examinations meeting the mandatory criteria of the current China BD standard. These examinations comprised: two EEG (electroencephalograph) recordings of at least $30 \mathrm{~min}$ performed 12$24 \mathrm{~h}$ apart demonstrating electrocerebral inactivity, the absence of intracerebral filling at the level of circle of Willis by TCD (transcranial Doppler) and apnoea testing [3]. The endpoint for the analysis was BD confirmed by EEG and TCD $[3,4]$.

\section{Statistical analysis}

All analyses were carried out using SPSS 13.0 for Windows (SPSS, Chicago, IL). The two-tailed significance level was set at $\mathrm{P}<0.05$. Continuous variables were described as mean standard deviation, categorical factors as frequency and percentage. Pair wise comparisons were performed using Student's t-test and chisquare test as appropriate. We used ROC curve analysis to identify the cutoff to dichotomise continuous variables of interest, or the estimated score that increased the sum of sensitivity and specificity to the highest amount. Final multivariable logistic models retained only factors found significant at the $\mathrm{P}<0.05$ levels using a stepwise forward/backward selection method. We then used these predictive probabilities to estimate the area under the ROC.

\section{Results}

Between May 1, 2015 and April 30, 2016, we assessed 104 patients. Table 1 summarizes baseline characteristics of our study population. 29 patients (27.9\%) were identified as brain death within 3-5 days after spontaneous respiratory arrest, $50(48.1 \%)$ were identified between 5-7 days, and 25 (24\%) were identified after 7 days.

The analysis results show that there were significant difference between the three groups of age, the difference was statistically significant $(\mathrm{P}<0.05)$; No obvious difference was found between gender distribution, there was no statistically significant difference $(\mathrm{P}$ $>0.05$ ); In clinical characteristics, diagnosis, swirl sign, hydrocephalus and subarachnoid hemorrhage has no difference between the three groups, there was no statistically significant difference $(\mathrm{P}>0.05)$; In other clinical indicators, distribution exists obvious difference between the three groups, the difference was statistically significant $(\mathrm{P}<0.05)$.

According to the results of univariate logistic regression analysis of gender, age, diagnosis, corneal reflection, cough reflex, swirl sign, hydrocephalus and subarachnoid hemorrhage had no obvious effect on brain death, and the pupil shape, pupil light reflex, motor response to pain, cisterna ambiens, cerebral hernia and intraventricular hemorrhage were associated with BD (Table 2 and Figure 1).

Multivariable logistic regression analysis showed that absent pupil light reflex $(\mathrm{OR}=7.43,95 \% \mathrm{CI} 1.62-33.99, \mathrm{P}=0.010)$, anisocoria or bilaterally dilated pupil size $(\mathrm{OR}=6.25,95 \% \mathrm{CI} 1.21-32.22, \mathrm{P}=$ $0.028)$, absent motor response $(\mathrm{OR}=15.89,95 \% \mathrm{CI} 3.04-82.93, \mathrm{P}=$ $0.001)$, absent cisterna ambiens $(\mathrm{OR}=11.96,95 \% \mathrm{CI} 1.59-89.78, \mathrm{P}$ $=0.016)$ and present of brain herniation $(\mathrm{OR}=16.28,95 \%$ CI 2.25 $117.73, \mathrm{P}=0.006)$ on brain CT scan were associated with $\mathrm{BD}$ within 7 days (Table 3 and Figure 2).
Table 2: Association between baseline characteristics and progression to BD.

\begin{tabular}{|l|l|l|l|}
\hline Factors & Units & OR (95\% Cl) & P \\
\hline Gender & Vs. male & $1.19(0.68-3.76)$ & 0.769 \\
\hline Age (years) & Continuous & $1.01(0.97-1.05)$ & 0.600 \\
\hline Diagnosis & 60 -vs. <60 & $2.76(0.44-17.64)$ & 0.281 \\
\hline Pupil shape & Vs. Hemorrhagic & $0.84(0.34-2.11)$ & 0.713 \\
\hline Pupil light reflex & Vs. normal & $5.08(1.62-15.94)$ & 0.005 \\
\hline Corneal reflex & Vs. sensitive & $4.79(1.65-13.89)$ & 0.004 \\
\hline Cough reflex & Vs. sensitive & - & 0.970 \\
\hline Motor response to pain & Vs. yes & $3.82(0.44-33.41)$ & 0.226 \\
\hline Cisterna ambiens & Vs. sensitive & $6.46(2.00-20.89)$ & 0.002 \\
\hline Swirl sign & Vs. normal & $5.62(1.52-20.77)$ & 0.001 \\
\hline Cerebral hernia & Vs. no & $1.19(0.46-3.02)$ & 0.723 \\
\hline Intraventricular hemorrhage & Vs. no & $5.31(1.41-19.97)$ & 0.014 \\
\hline Hydrocephalus & Vs. no & $3.77(1.24-11.49)$ & 0.020 \\
\hline Subarachnoid hemorrhage & Vs. no & $0.84(0.23-3.08)$ & 0.792 \\
\hline & & $0.63(0.25-1.62)$ & 0.341 \\
\hline
\end{tabular}

Table 3: Multiple logistic regressions.

\begin{tabular}{|l|l|l|l|}
\hline Factors & Units & OR $(\mathbf{9 5} \% \mathbf{C l})$ & $\mathbf{P}$ \\
\hline Pupil size & Vs. normal & $6.25(1.21-32.22)$ & 0.028 \\
\hline Pupil light reflex & Vs. sensitive & $7.43(1.62-33.99)$ & 0.010 \\
\hline Motor response to pain & Vs. sensitive & $15.89(3.04-82.93)$ & 0.001 \\
\hline Cisterna ambiens & Vs. normal & $11.96(1.59-89.78)$ & 0.016 \\
\hline Cerebral hernia & Vs. normal & $16.28(2.25-117.73)$ & 0.006 \\
\hline Intraventricular hemorrhage & Vs. normal & $1.77(0.03-98.26)$ & 0.781 \\
\hline
\end{tabular}

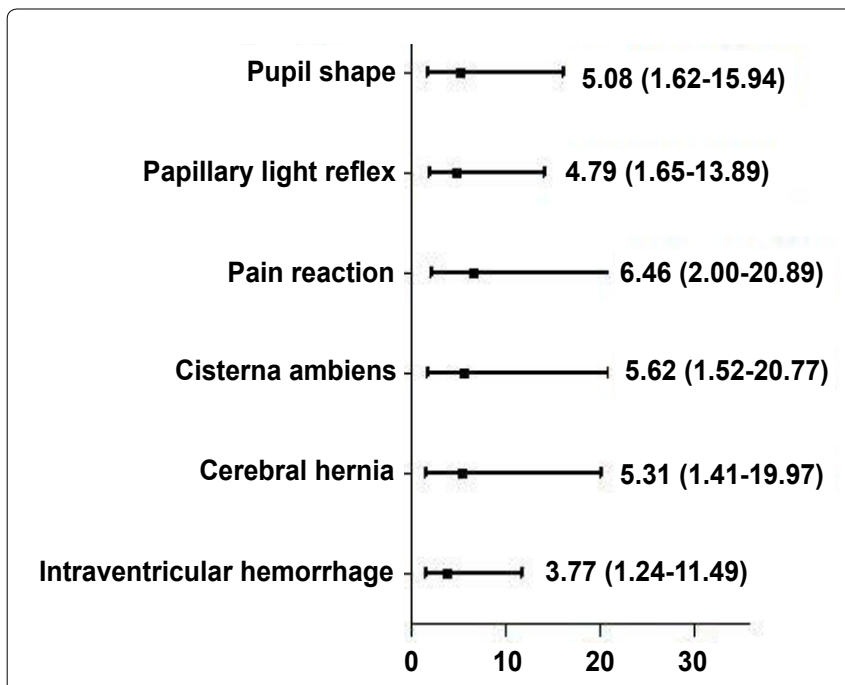

Figure 1: Univariate logistic regression OR value distribution.

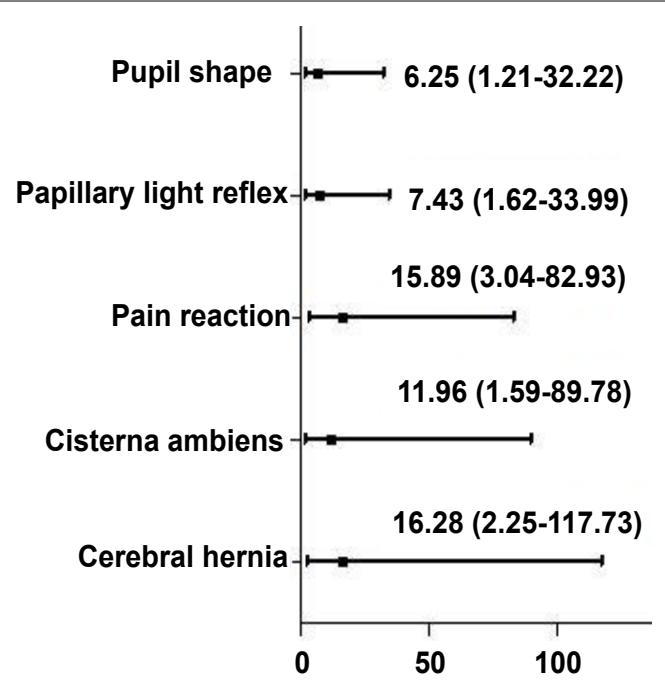

Figure 2: Multivariate logistic regression $O R$ value distribution. 
Table 4: The BD-N score

\begin{tabular}{|l|l|l|}
\hline Variable & Normal & Abnormal \\
\hline Pupil light reflex & 0 & 1 \\
\hline Pupil size & 0 & 1 \\
\hline Motor response to pain & 0 & 1 \\
\hline Cisterna ambiens & 0 & 1 \\
\hline Brain herniation & 0 & 1 \\
\hline
\end{tabular}

Table 5: Probability of BD after spontaneous respiratory arrest in acute neurocritical patients

\begin{tabular}{|l|l|l|}
\hline Score & $\mathbf{5 7 d}$ group $(\mathbf{n}=\mathbf{7 9})$ & $\mathbf{7} \mathbf{7 d}$ group $(\mathbf{n}=\mathbf{2 5})$ \\
\hline 1 & $0(0)$ & $3(100 \%)$ \\
\hline 2 & $2(28.6 \%)$ & $5(71.4 \%)$ \\
\hline 3 & $10(66.7 \%)$ & $5(33.3 \%)$ \\
\hline 4 & $19(73.1 \%)$ & $7(26.9 \%)$ \\
\hline 5 & $48(90.6 \%)$ & $5(9.4 \%)$ \\
\hline
\end{tabular}

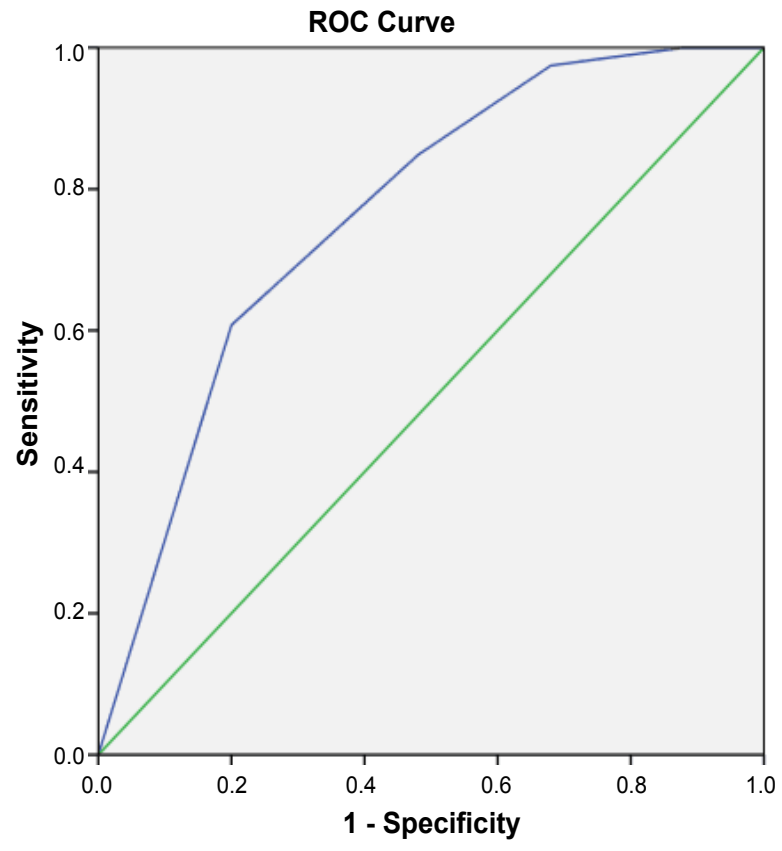

Figure 3: The area under the curve for the score.

We use the time of spontaneous respiration arrest as zero point, and limit the brain death time to 7 days according to the fundamental theory of brain injury and current BD judgment clinical practice (detailed statement in the discussion). We created the BD-N score (Table 4) to predict the chance of brain death within 7 days after spontaneous respiration arrest on the basis of odds ratios for every variable, assigning 1 points for absent pupil light reflex, anisocoria or bilaterally dilated pupil, absent motor response, absent cisterna ambiens and present of brain herniation (Table 4). The probability of BD within 7 days after spontaneous respiration arrest increased as the score increased (Table 5). The area under the curve for the score was 0.81 (95\% CI 0.75-0.87) for prediction of BD within 3-5 days, 0.77 (0.70-0.84) for BD between 5 and 7 days, and $0.76(0.69-0.83)$ for death after 7 days (Figure 3). Compared with a score of 1-2, a score of 3-5 had a sensitivity of $72 \%$; a score of 3 or more translates into an $81.9 \%$ probability of $\mathrm{BD}$ within 7 days (positive predictive value) whereas a score of $1-2$ translates into an $80 \%$ probability of BD after 7 days (negative predictive value).

\section{Discussion}

The clinical assessment of a patient suspected of BD is clearly defined in current guidelines, following these guidelines; the clinicians can feel confident of the declaration [6]. Early estimates have placed the prevalence of $\mathrm{BD}$ among patients with acute brain injury at $10 \%$ [7,8]. Meanwhile the China people accept the concept of brain death, progressively. The quality of donation after brain death (DBD) organs is concerning, since the DBD is becoming the main organ donation types in China.

Neurocritical patients was defined as an apneic patient with deep coma and GCS score of 3 [9]. Absent brain stem reflexes illustrate brain stem function impaired, once brain stem function is lost, respiration arrest first and the heart soon thereafter [10]. So identifying neurocritical patients who could progress to $\mathrm{BD}$ is particularly important at the acute phase before cardiac arrest. Therefore, neurocritical patients become the common candidates for deceased organ donation in China.

$\mathrm{BD}$ is a result of an unrelenting acute brain injury, the brain edema is the secondary brain injury and the main cause of $\mathrm{BD}$ [10]. A large number of studies have verified that brain edema occurred in 1-2 hours after brain injury, and reached the peak in next 24-48 hours, which lasted for 4-5 days. Spontaneous respiration arrest usually occurred during the brain edema peak. We use the time of spontaneous respiration arrest as zero point and limit the BD time to 7 days ( 2 days peak +5 days lasted) [11]. Other authors also advocated the time at 1 week so as to avoid the biased skewness of the deaths due to medical complications as a result of a long stay in the intensive care unit [3].

The severity of acute neurocritical patients remains a subjective notion and is currently based on the clinicians' experience taking into account all the clinical and radiological signs, which could predict a life-threatening condition for the patient [12]. A diagnosis of BD requires a massive brain or brain stem injury. The fundamental knowledge that the brain stem loses function is needed to understand the neurology of brain death, typically progressing from a hemispheric lesion to a brain stem lesion [10]. Multiple independent risk factors predicting the outcome of patients with TBI (traumatic brain injury) have been identified; the most widely accepted ones being age, GCS score, pupil size and reactivity, motor response to pain and CT scan findings, but those risk factors have not yet been identified as predictors of $\mathrm{BD}$ after spontaneous respiration arrest in acute neurocritical patient $[1,10,12]$. Based on this, we designed a prospective observational study to screen predictive factors for brain death.

There were 5 significant predictors of outcome from the multivariate analysis that were incorporated into the $\mathrm{BD}-\mathrm{N}$ score (a neurological scoring system). These predictors included 3 neurological variables focused on brain stem function and 2 cranial imaging variables focused on hemispheric lesion. In our study, absent pupil light reflex, anisocoria or bilaterally dilated pupil (pupil size) and absent motor response (motor response to pain) were associated with progression to $\mathrm{BD}$ in acute neurocritical patients in our study. Pupil size and reactivity were found to be strong predictors of survival and functional outcome; the best outcome is reportedly found in patients with bilaterally reactive pupils, and the worst was seen in patients with bilaterally fixed and dilated pupils. These were consistent with our study results, but these studies didn't design to predict $\mathrm{BD}[1,2]$. Several studies had identified the pupil reflex as one predictor of $\mathrm{BD}[10,13]$, but the pupil size and motor response to pain have not yet been identified as predictors of BD in acute neurocritical patient. However, Healey, et al. advocated the motor response to pain as the best choice because they considered it has the greatest impact on outcome prediction and simplified determination by avoiding misinterpretation in evaluating eye and verbal response subscores [14]. A GCS score of 3 is associated with an extremely high mortality rate, with some researchers suggesting that there is no reasonable chance of survival [2]. In our study, the patients included is GCS score of 3-5, identification of patients with a reasonable chance of survival within the GCS 3 collective is of overriding importance, especially the probability of progression to $\mathrm{BD}$ after spontaneous respiration arrest. We only choose specially appointed factors associated with brain stem function ( 5 to 7 times the drug's elimination half-life in hours and allow that time to pass before clinical examination is performed) because there are some observation misunderstandings on some reflections, such as the examiner must pay close attention because the 
corneal reflex may still be present as a subtle blink response with only a movement of the eyelashes. Such a mistaken observation may lead to further waiting and even loss of a potential organ donor due to cardiac arrest.

Brain stem reflexes have been a long sought model to predict the prognosis of patients with severe brain injuries. However, the major limitation of this type score is its assessment in the patients who are either sedated or under the influence of drugs or intubated for airway protection. Based on this limitation, the rational approach for prognostication of neurocritical patients is the utility of scoring system including the morphological criteria on radiological imaging. Among the current armamentarium, Magnetic resonance studies are basically limited for detecting white matter changes in the later phase of brain injury [15]. Meanwhile the head CT scan can be imaged very early after brain injury, so the CT imagine of brain injury became the rational approach for prognostication of neurocritical patients. However, in the patients with anoxic-ischemic encephalopathy who eventually fulfill brain death criteria, cerebral edema or marked hypodensities in thalami, caudate nuclei, and basal ganglia are typically seen on a follow-up CT scan. Determining brain death in a patient with repeatedly normal CT scans is never acceptable.

A scoring system based on CT imaging holds the greatest promise [12]. Current studies show absence of intracranial pathologies certainly adds up the odds for better outcome. Moreover, the role of individual characteristics within the model such as status of the cisterns, midline shift (MLS), subarachnoid hemorrhage (SAH), and mass lesions were associated with the outcome of the patients have already been detailed and verified [12]. Previous studies have verified the role of CT scores in predicting the mortality of patients following TBI $[10,13]$. Abnormalities on CT scan include a large mass with brain tissue shift (midline shift), multiple hemorrhagic lesions, diffuse cerebral edema with obliteration of basal cisterns, or traumatic SAH. Our study clearly corroborates the validity of cisterna ambiens and brain herniation in its ability to predict early mortality in acute neurocritical patients. We are furthermore able to show that cisterna ambiens and brain herniation (include midline shift) had a significant impact on the outcome of these patients. In a cohort study, Munakomi S, et al. clearly show that status of the cisterns, presence of midline shift and traumatic SAH on CT scan had a significant impact on the outcome of these patients [12]. The midline shift is the direct signs of brain herniation, own the same meaning with brain herniation used in our study. Cisterna ambiens is one of the intracranial cisterns, located around the brain stem; it reflects the brainstem compression or impaired status, directly. A drawback of the CT scoring model is the issue of inter-observer bias. Studies have shown that there is a significant difference in scoring of the CT image among trained radiologist as well $[10,16]$. Such differences in CT film read out may translate into modified treatment algorithms, which in itself can change the outcome measures. We tried to nullify this with validation of the score from two trained radiologist and inclusion of the highest score in the data [16].

These findings could form the basis of a simple score usable at the bedside to help clinicians identify patients likely to progress to BD. Nearly, all the patients in this study were identified by neurosurgeon after irreversible cessation of spontaneous respiration that is the interval we consider as being the acute phase during which our score would be applicable. The determination of BD is far more detailed than is usually assumed. Technical tests have been developed to support the clinical diagnosis of $\mathrm{BD}$ and can demonstrate absent blood flow to the brain or absent electrical activity of the cortex. Consequently, for prognostication purposes, this study recommends the use of a set of specific brain stem reflexes and imaging characteristics from the existing CT classification systems alone. In the future, it will therefore be important to develop prognostic models that are applicable for the majority of patients.

\section{Acknowledgements}

Guixing $\mathrm{Xu}$ and Jing Zhao participated in research design, performance of the research, and writing of the paper. Ping Xu participated in research design, performance of the research, and data analysis. Zhiyong Guo and Xiaoshun He participated in research design and writing of the paper. The authors have no conflict of interest. This study was supported by the Guangdong Provincial Key Laboratory on Organ Donation and Transplant Immunology(2013A061401007) and Guangdong Provincial International Cooperation Base of Science and Technology (Organ Transplantation)(2015B050501002).

\section{References}

1. Hoffmann M, Lefering R, Rueger JM, Kolb JP, Izbicki JR, et al. (2012) Pupi evaluation in addition to Glasgow Coma Scale components in prediction of traumatic brain injury and mortality. Br J Surg 99: 122-130.

2. Chamoun RB, Robertson CS, Gopinath SP (2009) Outcome in patients with blunt head trauma and a Glasgow Coma Scale score of 3 at presentation. J Neurosurg 111: 683-687.

3. Galbois A, Boëlle PY, Hainque E, Raynal M, Cazejust J, et al. (2013) Prediction of evolution toward brain death upon admission to ICU in comatose patients with spontaneous hemorrhage using simple signs. Transpl Int 26: 517-526.

4. He X, Xu G, Liang W, Liu B, Xu Y, et al. (2015) Nomogram for predicting time to death after withdrawal of life-sustaining treatment in patients with devastating neurological injury. Am J Transplant 15: 2136-2142.

5. Koivunen RJ, Satopaa J, Haapaniemi E, Strbian D, Meretoja A, et al. (2014) Predictors of early mortality in young adults after intracerebral hemorrhage. Stroke 45: 2454-2456.

6. Wijdicks EF, Rabinstein AA, Manno EM, Atkinson JD (2008) Pronouncing brain death: contemporary practice and safety of the apnea test. Neurology 71: $1240-1244$.

7. Kramer AH, Zygun DA, Doig CJ, Zuege DJ (2013) Incidence of neurologic death among patients with brain injury: a cohort study in a Canadian health region. CMAJ 185: E838-E845.

8. Kompanje EJ, de Groot YJ, Bakker J (2011) Is organ donation from brain dead donors reaching an inescapable and desirable nadir? Transplantation 91: $1177-1180$

9. Dodek P (2003) Mandatory reporting of "imminent" death to identify organ donors: history, controversy, and potential solutions. Can J Anaesth 50: 955960.

10. Wijdicks EF (2015) Determining Brain Death. Continuum (Minneap Minn) 21: 1411-1424

11. Kinoshita K (2016) Traumatic brain injury: pathophysiology for neurocritical care. J Intensive Care 4: 29

12. Munakomi S, Bhattarai B, Srinivas B, Cherian I (2016) Role of computed tomography scores and findings to predict early death in patients with traumatic brain injury: A reappraisal in a major tertiary care hospital in Nepal. Surg Neurol Int 7: 23.

13. Humbertjean L, Mione G, Fay R, Durin L, Planel S, et al. (2016) Predictive factors of brain death in severe stroke patients identified by organ procurement and transplant coordination in Lorrain, France. Transpl Int 29: 299-306.

14. Healey C, Osler TM, Rogers FB, Healey MA, Glance LG, et al. (2003) Improving the Glasgow Coma Scale score: motor score alone is a better predictor. J Trauma 54: 671-678.

15. Uchino Y, Okimura Y, Tanaka M, Saeki N, Yamaura A (2001) Computed tomography and magnetic resonance imaging of mild head injury - Is it appropriate to classify patients with Glasgow coma scale score of 13 to 15 as "mild injury"? Acta Neurochir (Wien) 143: 1031-1037.

16. Havill JH, Sleigh JW, Davis GM, Chatterton BJ, Gilbert KW, et al. (2001) Observer error and prediction of outcome - Grading of head injury based on computerised tomography. Crit Care Resusc 3: 15-18. 